\title{
A CASE OF MEGADUODENUM WITH MULTIPLE ANOMALIES OF THE ILEUM
}

BY

\author{
RICHARD H. HUNTER, M.D., M.Ch., Ph.D. \\ (From the Department of Anatomy, Queen's University, Belfast.)
}

A chronic dilatation of the duodenum is sometimes found in patients suffering from diseases of the stomach, gall-bladder or pancreas. In such cases the gut wall is thinner than normal. Such a condition must be differentiated from that of megaduodenum, in which with a dilatation the muscle wall of the duodenum is hypertrophied; a condition similar to megacolon.

Cases of megaduodenum are rare; Birnbaum and Blacker ${ }^{1}$ make no reference to it, and in the literature $I$ have been able to find only three instances of it. One of these occurred in a child of four and a half years, and is described by Downes ${ }^{2}$; another occurred in a ' young adult' and is described by Corwin ${ }^{3}$; and a third case is referred to by Corwin as having been seen by W. J. Mayo. The condition is stated by Corwin to be congenital in origin, probably implying that it is the result of some defect in the development of the gut wall; but Downes states that ' it is much more probable that the gut is normal at birth, and that the distension and hypertrophy result from constriction at some point, usually the duodeno-jejunal junction.'

\section{Pathological report of case.}

The body from which this specimen was obtained was that of a still-born male infant which weighed $7 \mathrm{lb}$. It had a slightly distended abdomen. On opening the abdominal cavity several loops of greatly dilated intestine presented themselves. Those loops from the upper abdomen proved to be dilated duodenum (Fig. 1), and those from the lower abdomen were dilated parts of the ileum. The duodenal dilatation was crossed by the superior mesenteric vessels, but these produced no narrowing, although the appearance of the photograph would suggest a constriction at the site of the crossing. The pyloric canal was dilated, and there was no sphincter separating pyloric stomach from duodenum. The cardiac part of stomach was normal. The duodenal-jejunal flexure was normal in its position and appearance, and there was nothing in the nature of a mesenteric band or other form of constriction such as inflammatory adhesions or enlarged mesenteric glands, as suggested by Downes, to cause the condition. The liver and gall-bladder presented no unusual appearances. The common bile duct was distended with bile and was constricted at the duodenal opening. The jejunum throughout its whole length was definitely normal in respect of size, form and structure. Three areas of ileum, however, presented dilatations similar to the dilatation of the duodenum. These dilated parts were connected together by short lengths of fibrous cord-like bands, which lay along the free edge of the mesentery, in the position usually occupied by the gut (Fig. 2). At one point in the fibrous cord there was a short length of normal gut. The large gut was normal in all its appearances and at the stage of development normally found in new-born infants. There was little or no meconium in the large gut, and there was 
no content in any of the ileal cyst-like dilatations. As the specimen was examined within twenty hours of death it is certain that there had not been any absorption of fluid content.

The microscopic examination of one of the connecting bands showed it to consist entirely of fibrous tissue, in which there was no trace, as shown by differential staining, either of glandular or muscular tissue to suggest intestine. The fibrous character of the cord was so complete that it is difficult to say whether it represents a primary condition with a total absence of muscle and epithelium differentiation, or whether it is a secondary change with a degeneration of muscle and epithelium. The dilated portion of the ileum, on microscopic examination (Fig. 4) showed hypertrophy of the muscle walls, the longitudinal layer of muscle being more thickened than the circular. The two layers of muscle were not in close contact, as

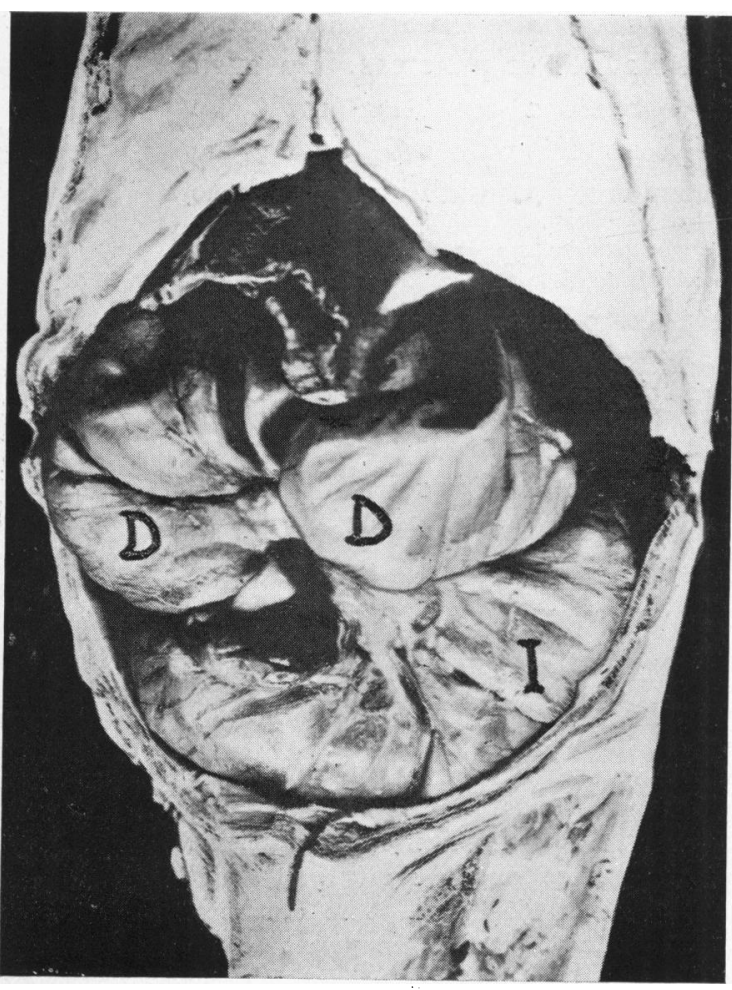

FIG. 1.-Photograph of the dilated loops of - duodenum and ileum which presented on opening the abdomen.

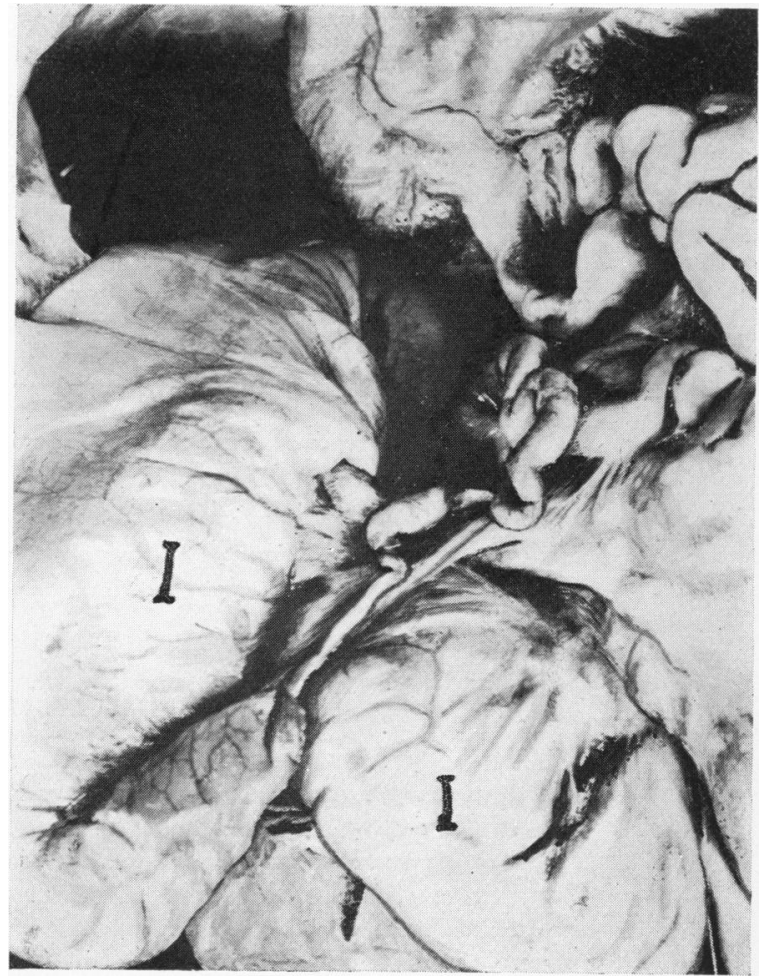

FIG. 2.-Photograph of the ileal dilatations with a band connecting one of them to a short portion of normal ileum.

in the normal gut, but were separated from one another by a well developed layer of fibrous connective tissue. The mucosa was poorly developed and formed a thin layer around the inner surface of the muscular tube. Peyer's lymphatic patches were arranged in thin elongated diffused areas. The microscopic characters of the duodenum (Fig. 5) showed a similar hypertrophy, again particularly of the longitudinal wall, and an attenuated layer of mucosa. The two muscle layers, as in the ileum, were separated from one another by a thick layer of connective tissue, and the submucosa consisted of a wide band of strong fibrous tissue, exceeding in thickness that of the circular muscle layer, and in it there was no indication of any œdematous changes. The blood vessels of the sub-mucosa of the dilated duodenum and ileum were greatly enlarged. Careful examination of the sections was made for the myenteric plexus but mere traces only of it could be found. 
The ætiology of atresia of the gut is well known. It is due to a failure of the gut to form a lumen. Tandler ${ }^{4}$ and others have shown that stenosis is the normal condition of the gut at the fifth week of embryonic life. The condition is brought about by the rapid proliferation of the primary epithelial cells which line the simple tube, and the cells quickly occlude the tube. Some weeks later in development the lumen of the gut reappears, and is formed by a fusion of certain vacuoles or cleft-like spaces which appear in the solid cord of cells. The persistence of the solid tube stage of the gut, with failure to differentiate and form the mucosa and muscle layers, results in the formation

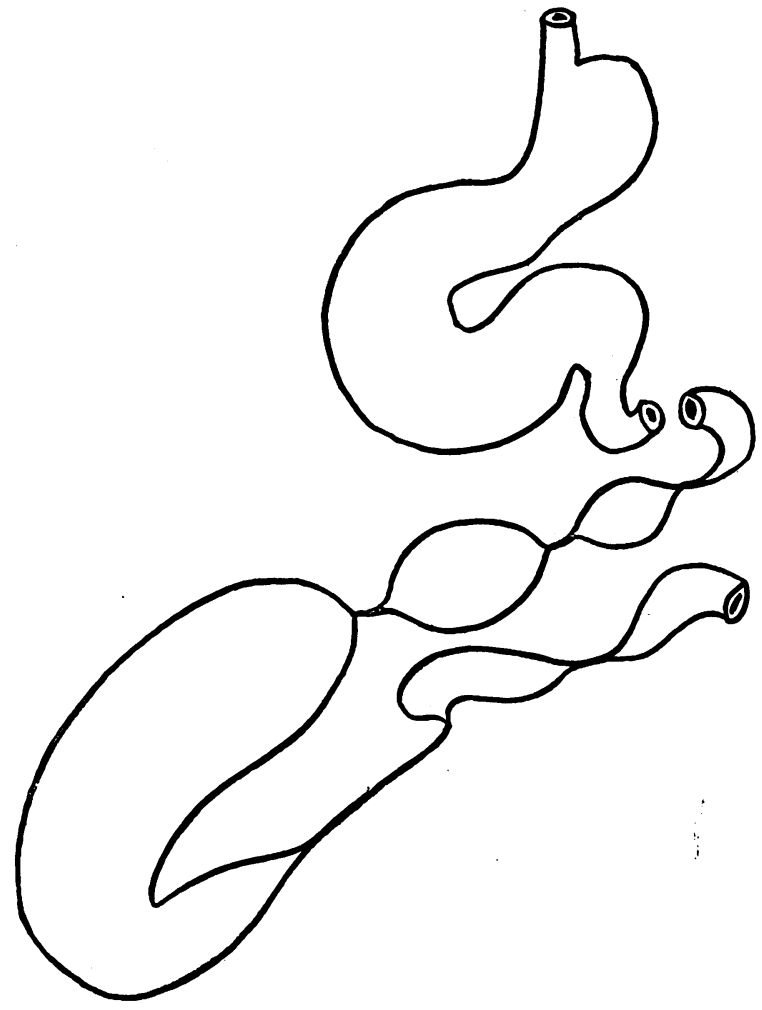

Fig. 3.-Diagram to show the general arrangement of the atresias and the dilatations of pyloric canal, duodenum, and ileum. The normal jejunum is not shown.

of bands of fibrous issue. Atresia is relatively common. Of 392 cases collected by Davis and Poynter ${ }^{5}$ the obstruction was located 134 times in the duodenum, 60 times in the jejunum, 101 times in the ileum and cæcum, and 39 times in the colon.

It is more difficult to find an explanation for the dilated portions of the gut. These may be primary unconditioned hypertrophies, or they may be secondary conditions caused by an attempt of the gut to overcome the obstruction of the atresia. The dilatation and hypertrophy of the duodenum would suggest that the condition is a primary one, as no obstruction was present at the duodeno-jejunal junction, and the jejunum was normal from 
that point to the position of the first fibrous area. Another point in support of this view is that the cardiac area of the stomach was normal in development, yet the pyloric canal was dilated, and there was no evidence of a pyloric sphincter. The probability of an incoordination between the sympathetic and parasympathetic nerve supply might be considered as the causative factor, as suggested for the causation of megacolon ${ }^{6}$. Dilatation of the colon can be produced experimentally by section of the sympathetic nerves of the gut $^{7}$, but no evidence has been brought forward that hypertrophy, which is the essential part of the change, can be produced by this means. Then again, the work of $\mathrm{Abel}^{8}$ makes it clear that development of

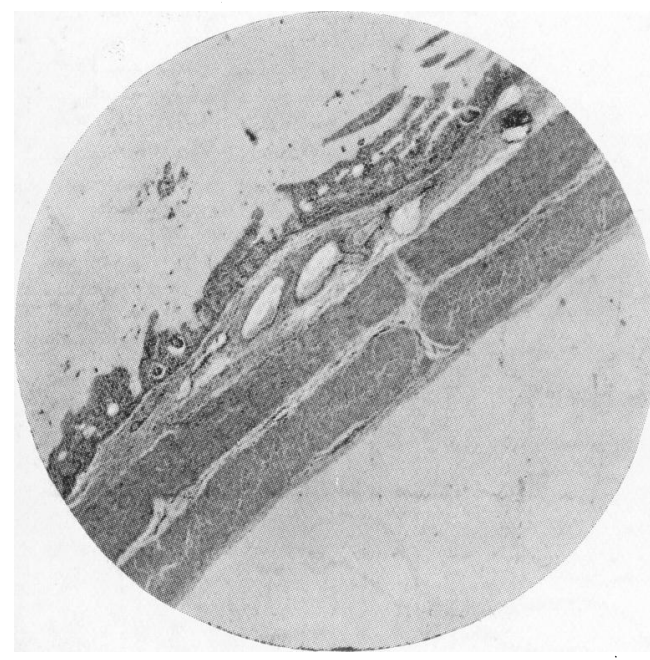

Fig. 4.-Microphotograph of the wall of the dilated ileum. Note the thickening of the longitudinal layer of muscle; the thick band of fibrous tissue between it and the circular layer of muscle, also the thin layer of mucosa.

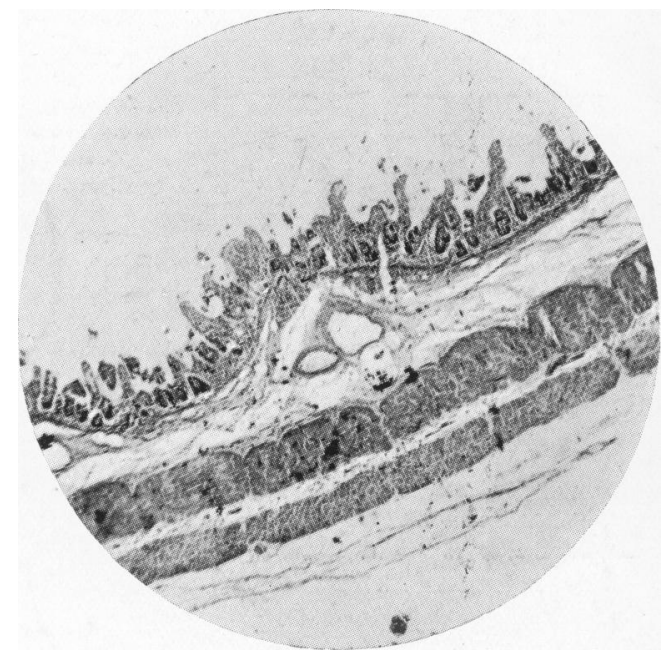

FIG. 5.-Microphotograph of the dilated duodenum. The thickening of the fibrous layer between the thickened circular and longitudinal muscles is very marked, as is also the sub-mucous fibrous layer.

muscle progresses normally in the absence of nerve impulses. It has also been shown by Tower ${ }^{9}$ that the influence of the sympathetic nervous system is not necessary for the growth of muscle in the kitten.

A nervous origin for the dilatation and hypertrophy of the gut in the specimen here described, is not therefore considered as a likely explanation of the condition.

\section{REFERENCES.}

1. Birnbaum, R., \& Blacker, G., Malformations and Cong. Dis. of the Fœetus, London, 1912.

2. Downes, W. A., Ann. Surg., Philad., 1917, LXVI, 289.

3. Corwin, R. W., Colorado Med., Denver, 1915, XII, 138.

4. Tandler, A., Morph. Jahrb., 1902, XXXIX, 187.

5. Davis, D. L., \& Poynter, C. M. N., Surg. Gynec. Obst., Chicago, 1922, XXXIV, 35.

6. Illingworth, C. F., \& Dick, B. M., Surgical Path., Edinb., 2nd Edt, 1932, 460.

7. Adamson, A. D., \& Aird, I., Brit. J. Surg., Bristol, 1932, XX, 229.

8. Abel, Williamina, J. Anat., London, 1912, XLVII, 47.

9. Tower, S. S., Annual Report of the Director of the Department of Embryology, Carnegie Institute of Washington, 1932, 16. 